\title{
Artikel
}

Markus Steinmayr*

\section{Poetik, Provokation, Lektüre. Björn Höcke und Rolf Peter Sieferle im Kontext}


Abstract: Die vorgelegten Überlegungen präsentieren anhand von Sieferles Finis Germania und Höckes Nie zweimal in denselben Fluss Überlegungen zur kulturellen und politischen Diskursstrategie der Neuen Rechten. Die Strategie wird anhand der Auseinandersetzung mit den Figuren des Lesens, des Umgangs mit Büchern und anhand des Umgangs mit Gegenständen und Figuren der Bildung rekonstruiert.

Keywords: Neue Rechte; Bildung; Autodidakt; Autobiographie; Repräsentation; Theatralität der Neurechten

*Markus Steinmayr, Universität Duisburg-Essen - Campus Essen, Essen, Nordrhein-Westfalen GERMANY

"Die wahre Liberalität", schreibt Goethe, „ist Anerkennung." 1 Goethe weist damit auf einen Prozess hin, der für das Verständnis unserer politischen Gegenwart wesentlich ist. Goethe markiert nämlich einen eminent wichtigen Zusammenhang zwischen dem, was ein Einzelner denkt und tut oder tun will, und denjenigen gesellschaftlichen Prozessen, die diese Tätigkeit oder dieses Denken zur Kenntnis nehmen, anerkennen und ihnen damit Sinn verleihen. Erst so, möchte man meinen, macht Liberalität Sinn. Eine Liberalität ohne Anerkennung, also ohne einen zwischen Individuum und Gesellschaft vermittelnden Prozess, bleibt resonanzlos. Prozesse dieser Art sind im besten Sinne Kommunikation. Dies gilt in besonderer Weise für den Umgang mit Meinungen, die nicht die eigenen sind oder die die Grenzen dessen, was man meinen kann, überschreiten. Der Umgang mit dem Anderen der eigenen Meinung bringt diesen Anerkennungsprozess an seine Grenzen. Das gilt insbesondere für den Umgang mit der Neuen Rechten. Die Anerkennung setzt einen Raum der Öffentlichkeit voraus, in dem die Prozesse stattfinden. Es geht also darum, die Texte der Neuen Rechten aus ihren Zirkeln zu lösen und sie in die Öffentlichkeit der Analyse zu bringen. Anerkennung, auch dies wusste Goethe sehr genau, bedeutet gerade nicht immer Bestätigung.

Ohne Goethe zu erwähnen, schreibt Armin Nassehi in Bezug auf seinen Briefwechsel mit Götz Kubitschek, dass „zu einer solchen Art von Liberalität" auch gehöre „mit Leuten ins Gespräch zu treten, deren Position man nicht teilt." ${ }^{2}$ Als Nicht-Soziologe trete ich im Anschluss an Nasse-

1 Goethe 2005 [o. D.], S. 872.

2 Nassehi 2015, S. 299. his Überlegungen ins Gespräch mit anderen Positionen durch das Lesen. Ich lese aber nicht nur die Rechten selbst, sondern auch, was und wie Rechte lesen oder lesen sollen.

So heißt es von Götz Kubitschek, dass dieser "Homer im Original"3 gelesen habe. In seinem Buch Provokation spricht er von einem "Kosmos rechten Denkens" ${ }^{4}$, der vor allem ein Kosmos aus Texten und Lektüren ist. Es folgt unmittelbar danach eine Art neurechter Lesedidaktik, die "Romane sogar noch besser als theoretische Schriften" hält, um die "Suche nach dem rechten $M a \beta^{\prime \prime} 5$ zu ermöglichen. Konsequenterweise erfolgt dann im gleichen Verlag eine Publikation, die sich mit den „je prägenden Lektüren" ${ }^{\prime 6}$ von einflussreichen Figuren der Neuen Rechten beschäftigt.

Um die literarische Sozialisation ${ }^{7}$ im Sinne der Neurechten zu steuern, entwerfen Ellen Kositza und Carolin Sommerfeld einen Vorlesekanon für die neurechte Erziehung. Unter dem schlichten Titel "Vorlesen"8 machen die Ehefrauen um Götz Kubitschek respektive Helmut Lethen recht deutlich, dass „insbesondere durch das Vorlesen der Eltern"9 die Einübung im Umgang mit Büchern und dem Kanon ermöglicht wird. Dementsprechend umfasst das genannte Buch ca. 150 Leseempfehlungen.

Es gibt Handbücher, die einem vielleicht deshalb zeigen wollen, wie man mit Rechten reden soll. ${ }^{10}$ Es gibt aber nur vereinzelt Handreichun-

3 Kubitschek 2017, "Über den Autor".

4 Kubitschek 2017, S. 46.

5 Kubitschek 2017, S. 47.

6 Kositza/Kubitschek 2020, S. 9.

7 Vgl. Pieper 2010.

8 Kositza/Lethen 2019.

9 Pieper 2010, S. 108.

10 Leo/Steinbeis/Zorn 2017. 
gen, die darüber Aufschluss geben, wie Rechte lesen." Es gibt aufschlussreiche Dokumentationen, die über die longue durée rechten Denkens informieren. ${ }^{12}$ Angesichts der regen Publikationstätigkeit der Neuen Rechten ist eine Diskrepanz zwischen der häufig nur antizipierten Bedeutung von Büchern und der tatsächlichen Auseinandersetzung mit den Texten in der Öffentlichkeit festzustellen. Die Geschichtswissenschaft und die Literaturwissenschaft haben dieses Diskrepanzphänomen vor allem an Adolf Hitlers „Mein Kampf" aufgezeigt. ${ }^{13}$

Die Auseinandersetzung mit den Gedanken, Figuren, Bildern und narrativen Strategien, die in den Texten zirkulieren, dient dem Ziel, die Texte für eine Analyse zu öffnen und sie damit in ihrer Fiktionalität und Rhetorizität zu entlarven. Wenn man so will, geht es um ein Verfahren der Entsubstanzialisierung jener Substanzen, die die neuen Rechten und ihre Texte wie eine Monstranz vor sich hertragen: Volk, Heimat, Identität. Bei genauerer Analyse erweist sich diese Substanzialisierung als Wiederholung altrechter Themen, die unter den Bedingungen der Gegenwart scheinbar neu entdeckt werden, sich aber nur als Bricolage von Lektüren erweisen.

Auf eine gewisse Art und Weise Auslöser für diese Auseinandersetzung mit den mir bislang unbekannten Texten von Höcke und von Sieferle war die Lektüre von Heinrich Deterings "Was heißt hier ,wir'? Zur Rhetorik der parlamentarischen Rechten". ${ }^{14}$ Deterings Ausführungen sind insofern Vorbild für die hier vorlegten Überlegungen, als dass Deterings schmaler Band zeigt, wie nichtfiktionale Text- und Redesorten sich literaturwissenschaftlich lesen lassen, wenn man Politik und die politische Kommunikation auch als einen Umgang mit Texten und Tropen versteht. Sie sind ganz entscheidend für die Konstitution von Volk und Volksgemeinschaft, also von Fiktionen, die Zugehörigkeiten und Ausschlüsse imaginierbar werden lassen und regeln. ${ }^{15}$

In zustimmender Abgrenzung von Detering verstehen sich meine Ausführungen daher nicht als Beitrag zum Phänomen des Rechtspopulis- mus oder des Rechtsextremismus. Diese Auseinandersetzung ist von Detering und von anderen geleistet worden. Gleichwohl hinterlassen die Vorgänger*innen eine gewisse Lücke. Die Auseinandersetzung mit den Gedanken, Figuren, Bildern und narrativen Strategien, die in den Texten der Neuen Rechten zirkulieren, fehlt. Aber nur so kann man dem Ziel näherkommen, die Erzählund Darstellungsverfahren der Neuen Rechten zumindest in Ansätzen zu beleuchten. Es geht also um die Analyse der Wirksamkeit und Wirkmächtigkeit von Figuren, Lektüren und Selbstinszenierungen auf textueller Basis.

So erklärt sich die Auswahl der untersuchten Texte. Zum einen ist die Wahl auf Rolf Peter Sieferles Finis Germania gefallen, erstmals erschienen 2017. Dieser Text offenbart auf exemplarische Art und Weise die Formensprache der Neuen Rechten. Im Rekurs auf etablierte Formen der kulturellen und politischen Kommunikation in und mit Büchern wird das Buch Sieferles als Kultbuch der Neuen Rechten in Szene gesetzt. Es ist kein Buch, über das man diskutiert, sondern eines, mit dem man lebt. Ferner zeigen sich deutliche Anleihen bei etablierten Mustern der literarischen Formensprache (Tragödie) und bei Kritikmustern der rechten Tradition (Moderne als Verlust, vermeintliche Leere der politischen Repräsentation, Neujustierung der politischen Sprache).

Der zweite Text ist der Gesprächsband Nie zweimal in denselben Fluss. Das Buch ist die Dokumentation eines Gesprächs, das Björn Höcke mit Sebastian Hennig geführt hat. Es zeigt wiederum auf eine exemplarische Art und Weise die Selbstinszenierung neurechter Subjekte und ihres vermeintlichen Wissens. Dabei wird deutlich werden, dass dem Medium der Stimme und dem Nicht-Schriftlichen eine ganz besondere Bedeutsamkeit zukommt. Höckes politische Autobiografie erweist sich vor dem Hintergrund der Differenz Stadt/Land als Wiederkehr der Heimatkunst um 1900. Ferner spielt die Bildungsfigur des Autodidakten für die Selbstinszenierung Höckes eine prominente Rolle. ${ }^{16}$

11 Thomalla/Gladić 2021.

12 Fücks/Becker (Hg.) 2020.

13 Kiesel, 2014; Koschorke 2016; Kellerhoff 2015.

14 Detering 2019.

15 Wildt 2019.

16 Steinmayr 2021. 


\section{Rolf Peter Sieferle: Finis Germania}

Nimmt man Rolf Peter Sieferles Buch Finis Germania ${ }^{17}$ zum ersten Mal zur Hand, fällt sofort das Format dieses Buches auf: Ein kleines schmales Bändchen, gerade einmal 125 Seiten inklusive des Nachworts von Thomas Hoof. Über dem Zur-Hand-Nehmen dieses Buches schwebt der Skandal über das Erscheinen auf und Verschwinden von Bestsellerlisten. Diese Auseinandersetzung wird auch von Thomas Hoof in seinem Nachwort in voller Gänze ausgewalzt. ${ }^{18}$ Es handelt sich dabei um das Nachwort einer späteren Auflage des Sieferle'schen Buches im Landt-Verlag. Das Buch ist erstmals 2017 bei Antaios erschienen.

Ein unscheinbares Buch mit einem schwierigen Inhalt muss zunächst einmal in Umlauf gebracht werden. Das Format dieses Buches kommt dem sicherlich entgegen. Es passt in die Innentasche jenes Tweedsakkos, das Vertreter rechtspopulistischer Parteien sommers wie winters gerne tragen. Vom Verlag scheint es als eine Art Vademecum gedacht zu sein, es soll ein Handbrevier für den Rechtspopulisten werden, das er bei jeder Gelegenheit aus der Tasche ziehen kann. Das Format der Kaplaken-Reihe erscheint somit, wie Erika Thomalla und Mladen Gladić gezeigt haben, als Wiederkehr der Merve-Reihe. Beiden gemeinsam sind die Handlichkeit und die Attraktivität. ${ }^{19}$

Vergleicht man darüber hinaus den miniaturistischen Stil des Buches mit einem anderen Handbrevier, so liegt die perfide Strategie der Rechtspopulisten auf der Hand: Finis Germania soll die Minima Moralia der Neuen Rechten werden, also als Reflexion des beschädigten Lebens in der Migrationsgesellschaft fungieren.

Philipp Felsch hat die Minima Moralia als den Versuch Adornos bezeichnet, eine "neue Gebrauchsweise von Philosophie" ${ }^{10}$ zu etablieren. Was Sieferle intendiert, ist eine neue ,Gebrauchsweise' von Politik zu etablieren. Unter der Überschrift "Politiker und Intellektuelle" zeichnet Sieferle den positiv bewerteten Typus des „intellektuellen Politikers" (FG, 48). Er beschreibt dann

17 Sieferle 2019 [2017]. Hinfort wird Sieferles Text nach dieser Ausgabe mit der Sigle FG + Seitenzahl im Fließtext zitiert.

18 Grossarth 2017, Beyer 2017; Seibt et.al. 2017, Nutt 2017.

19 Thomalla/Gladić 2021.

20 Felsch 2015, S. 28. unter anderem die schriftstellerische Tätigkeit von Despoten und Diktatoren: "[S]elbst ein Stalin schämte sich nicht, seine Traktate und Reden als ,Gesammelte Werke' herausgeben zu lassen." (FG, 49.) Sieferle isoliert somit den Traktat-Stalin vom dichtenden Stalin, also den politischen Aktivisten vom Autor fiktionaler Texte. Beide kommen aber, darauf hat die slawistische Forschung hingewiesen, auf eigentümliche Art und Weise bei Stalin zusammen. Der Zweck wäre dann, den politischen Einsatz von Fiktionen in nicht-fiktionaler Literatur genauer zu untersuchen. ${ }^{21}$

Hitler, dem Sieferle bescheinigt, „Politiker und Programmatiker in einem" (FG, 50) gewesen zu sein, sei von dem Wunsch beseelt gewesen, als "Theoretiker zu gelten" (FG, 50). Die schreibenden Politiker von heute formulieren dagegen keine ,Theorien', sondern den "dritten Aufguß breitgewalzter Akademiethemen" (FG, 50). Für Sieferle ist diese Publikationstätigkeit von Politikern Ausdruck der Theorieunfähigkeit von Politik, die nunmehr auf das „Niveau des Kultur-Geschwätzes" (FG, 50) herabgesunken ist. Politische Kommunikation, so die Zeitdiagnose, ist Verfall eines „prinzipiell-ideologischen Charakters" (FG, 50) von Politik.

Die Frage nach der Funktion von Theorie im Spektrum des Rechtpopulismus legt den Vergleich mit Adornos Minima Moralia nahe. Die Minima Moralia gelten als Muster gesellschaftsthematisierenden und -kritisierenden Schreibens. Sie sind damit das Urmuster einer Gattung und formbildend. Gattungen, so lautet eine einschlägige Definition, sind ein "institutionalisiertes, auf sozialen Konventionen beruhendes textuelles Ordnungsmuster".22 Gattungen, aber auch Genres bilden also Schnittstellen zwischen Form und Gesellschaft.

Unter Genre versteht man "eine Gruppe von Texten mit ähnlichen Eigenschaften." ${ }^{23}$ Rüdiger Safranski hat vorgeschlagen, Finis Germania dem Genre der Nachtgedanken zuzuordnen. ${ }^{24}$ Die Ähnlichkeiten mit Edward Youngs Nachtgedanken in der englischen Literatur liegen tatsächlich auf der Hand. Bedeutsam beim Genre, Nachtgedanken' ist die Untersuchung der Sitten, ohne in die moralische Erziehung zu verfallen. "Gleichwie der Anlaß

21 Dobrenko 2011.

22 Zymner 2005, S. 261.

23 Ludwig 2017, S. 275.

24 Safranski 2017. 
zu diesem Gedichte", heißt es bei Young, "keine Erfindung, sondern eine wirkliche Geschichte war; so ward auch die darinn [sic] gebrauchte Methode dem Verfasser vielmehr durch das, was bey dieser Gelegenheit in seiner Seele von selbst vorgieng, vorgeschrieben, als überdacht, oder entworfen." 25

Es sind also Selbstexperimente im Denken, die wir in den Nachtgedanken vorfinden. Im Vergleich zu moralischen Erzählungen, argumentiert Young, ist "die Erzählung kurz, und die daraus entspringenden Sittenlehren machen den größten Theil des Gedichts aus". ${ }^{26}$ Ähnlich verhält es sich mit Sieferles Text. Der Text ist der Versuch, eine Sittenlehre der gegenwärtigen Bundesrepublik zu schreiben, eine Art neurechte Moralistik in den Untiefen des Liberalismus. Aus diesem Grunde finden sich bei Sieferle über den Text verstreut Bemerkungen zur Politik und zur Kultur, deren Beschreibung als Beschreibung der Sitten erscheint.

Texte wie die genannten bearbeiten die Schnittstelle von Ästhetik und Gesellschaft. Genau darum könnte es den Rechtspopulisten auch zu tun sein: nämlich mit Finis Germania die Schnittstelle zwischen Literatur und Gesellschaft, zwischen der literarischen Form und ihrer gesellschaftlichen Wahrnehmung neu und anders zu gestalten und damit eine ,neue Gebrauchsweise' rechten Denkens zu etablieren. Es sind in Max Czolleks Sinne „Blockbuster" ${ }^{27}$, die, breitenwirksam angelegt, dazu dienen, den "politischen Gegner" ${ }^{\prime 28}$ erkennbar werden zu lassen.

Während der Adorno lesende Linksintellektuelle, wie Philipp Felsch gezeigt hat, zum Stereotyp geworden ist, stellt sich das Bild des FinisGermania-Lesenden nicht so schnell ein. Wer soll dieser/diese Leser*in sein? Aufschluss hierüber gibt hier das Nachwort von Thomas Hoof. Nachdem in einem unerträglichen Ton die Debatte in den Qualitätsmedien beschrieben worden ist, die geschichtsrevionistischen Teile (Mythos VB) und die sieferle-kritisierenden Journalisten Grossarth, Hintermeier, Beyer als „zu einfältig" 29 beschrieben

25 Young 1768, S. 4.

26 Ebd.

27 Czollek 2020 [2018], S. 12.

28 Czollek 2020 [2018], S. 13.

29 Hoof, Thomas: Das ominöse "ominös" vor einer ominösen Zahl - und seine Folgen (Im Original in Großbuchstaben). Nachwort (FG, 115-123, hier 123). worden sind, um das Buch zu verstehen, schreibt Hoof in direkter Ansprache an die imaginierten ,Anderen' des Diskurses:

„Die dauernde Unterforderung Eurer Leser ist eine einzige Provokation. Und euer Meuteverhalten hat sich in eine geradezu brünstige Mitläufigkeit ausgewachsen, die auch weitergesteckte Ekelgrenzen mit Karacho überrennt. Eure Leser wenden sich nicht nur ab. Sie tun es mit Grausen. " (FG, 124.)

Die direkte Ansprache der Journalisten ist natürlich ein starkes rhetorisches Signal. Es wiederholt den Code Ihr/wir, Freund/Feind und konserviert die politische Affektlage des Zorns. Wie man aus der Rezeptionsästhetik lernen kann, rechnet jeder Text mit einem bestimmten/einer bestimmten Leser*in, der/die durch die Lektüre konstruiert wird. Der/die Leser*in als Subjekt der Lektüre ist eine, wie Gerhart von Graevenitz gezeigt hat, Erfindung des Textes selbst. ${ }^{30}$

Diese Phänomenologie der Lektüre lässt sich auch an der Konstruktion der imaginierten Leser*innenschaft von Rolf Peter Sieferles Finis Germania verdeutlichen. Der/die Leser*innen, wie es das Nachwort insinuiert, ist eine*r, der/die unterfordert und zornig ist. Im Gegensatz zu den professionellen Schreiber*innen und Leser*innen im Journalismus und in den Geistes- und Sozialwissenschaften, die Komplexität zunächst erzeugen, um sie dann zu reduzieren, sehnt sich der/ die angebliche Sieferle-Leser*in nach Kanälen, in denen er*/sie seinen/ihren Affekten freien Lauf lassen kann. Die politische Didaktik, die möglicherweise dahintersteckt, ist, die etablierten Wege der nicht simplifizierenden Komplexitätsreduktion zu verlassen und durch Affektpolitik bzw. durch eine Politik der Affekte zu ersetzen. ${ }^{31}$ Mir scheint, dass hier möglicherweise die kommunikative Logik der Sozialen Medien auf die Buchkommunikation übertragen wird. ${ }^{32}$ Die Logik, so könnte man sagen, bestünde darin, eine tendenziell ungeregelte Öffentlichkeit zu schaffen, in der alles gesagt werden darf, aber nichts geregelt werden muss. Die Kommunikationsökonomie der

30 Graevenitz 1973.

31 Lehmann 2019.

32 Vgl. zur Enthemmungslogik der neurechten Kommunikation und der daraus resultierenden Produktion von Halbwahrheiten: Gess 2021, insbesondere S. 86-101 und die Beiträge in Koch (Hg.) 2020. 
Neuen Rechten entspricht in genauer Weise der Kommunikationsökonomie der Sozialen Medien. ${ }^{33}$ Das sorgt im Buch selbst für einen gewissen Irritationseffekt.

So stiftet man Leser*innengemeinschaften, die aber programmatisch die Kommunikation mit divergierenden Meinungen verweigern. Es sind enthemmte Leser*innen. Der/die sich von angeblich simplifizierenden Wirklichkeitskonstruktionen des ,Mainstreams' abwendende Leser*in soll nun das Buch in die Hand nehmen und es als Brevier in der Tasche tragen. Es wird ein geradezu kultartiges Buch in diesem Nachwort konstruiert, das mit der Simplifizierung der Verhältnisse endgültig Schluss macht. Der Rechtspopulismus konstituiert so eine Leser*innengemeinschaft. ${ }^{34}$ Man kann diese Herstellung von Leser*innengemeinschaften als ,metapolitische' Strategie bezeichnen. Eine "zentrale Aufgabe metapolitischer Arbeit" besteht darin, wie es in Thor von Waldsteins Metapolitik heißt, "die in der öffentlichen Atmosphäre ständig präsenten Emotionen und Subjektivismen zu bündeln und einem sinnvollen Tätigkeitsgebiet zuzuführen". ${ }^{35}$ Es geht um den Angriff auf die, um es im Jargon des neurechten Gramsci-Ekkletizismus zu sagen, Hegemonie der politischen Emotionen.

Das Zweite, was auffällt, wenn man Rolf Peter Sieferles posthum erschienenes Finis Germania zur Hand nimmt, ist selbstverständlich der Titel. Will man den Titel "Finis Germania" ins Deutsche übersetzen, so ist die bisherige Rezeption schnell bei „Das Ende Deutschlands”, ein beliebtes Motiv der neurechten Apokalyptiker, das mit Sarrazins Deutschland schafft sich ab 2010 wieder in die Debatte eingeführt worden ist. Es gibt aus meiner Sicht zwei Möglichkeiten, den Titel zu lesen. Die erste Möglichkeit besteht darin, den Titel in den Modus einer Aussage zu überführen. Finis Germania bedeutete dann: Ende, Deutschland. Eine der Formen, das Ende in Szene zu setzen, ist die Tragödie. Bekanntlich bildet die Theorie der Tragödie das Herzstück der Aristotelischen Poetik. Seit Botho Strauß” Essay "Anschwellender Bocksgesang" kann man die Form der Tragödie mit guten Gründen als neurechte Form analysieren, Geschichte und Geschichten zu erzählen. ${ }^{36}$

33 Vogl 2021.

34 Vgl. nochmals Thomalla/Gladić 2021.

35 Von Waldstein 2019, S. 37.

36 Strauß 1993.
Aristoteles hat in seiner "Poetik" die Tragödie als die Gattung des Endes bestimmt. Bekanntlich ist die Tragödie die Nachahmung einer "in sich geschlossenen und ganzen Handlung". ${ }^{37}$ Die "Nachahmung von Handlung" 38 heißt es dann bekanntermaßen, ist der "Mythos", den er als "Zusammensetzung der Geschehnisse" 39 begreift. Wie aber kann man, eine in sich geschlossene und ganze Handlung' verstehen? Ein Ganzes, heißt es dementsprechend, ist etwas, "was Anfang, Mitte und Ende hat". ${ }^{40}$ Der Anfang ist da, er folgt "nicht mit Notwendigkeit" 41 auf etwas anderes, ihm, dem Anfang, folgt aber notwendigerweise alles Andere. Das Ende hingegen folgt immer etwas, dem Ende aber folgt nichts mehr. Der Mythos des Aristoteles ist das Gegenteil von Geschichte, wie sie die Historiografie entwickelt. Übertragen auf die verquere Geschichtslogik der Neuen Rechten bedeutet dies, dass die Entwicklung von Gesellschaften oder die Richtung, die diese nimmt, nur als Schicksal erscheint, also als etwas Unabänderliches, dem man nicht entrinnen kann.

Deswegen schreibt Sieferle auch in Bezug auf den Umgang mit dem Holocaust davon, dass der "Mythos eine Wahrheit [ist], die jenseits der Diskussion steht" (FG, 73). Es kann hier nicht darum gehen, die unerträgliche Erzählung von den Deutschen als Sündenböcken zu analysieren, die zum „immerwährenden Mythos werden, um ihre Schuld zu sühnen" (FG, 79). Das ist so aberwitzig und perfide, weil es gleichsam antisemitische Narrative aufnimmt, um diese in ihr Gegenteil zu verkehren: Früher waren es die ,Juden', die die Schuld abtragen mussten und auf Erlösung warteten, heute sind es die Deutschen. ${ }^{42}$ Damit einher geht die Erlösungsphantasie, doch nun endlich von der Schuld erlöst zu werden: durch Vergessen bzw. durch Verschiebung oder durch Inversion von Täter-/Opferrollen. Sieferle perpetuiert hier nur den "deutschen Opfermythos"43, der ja bekanntlich zu den "Gründungsmythen der Bundesrepublik"44 gehört.

37 Aristoteles 1982 S. 25.

38 Aristoteles 1982, S. 19.

39 ebd.

40 ebd.

41 ebd.

42 Vgl. hierzu die Bemerkungen Czolleks (2020 [2018], S. 175ff.) zur Funktion der Ashaver-Legende.

43 Salzborn 2020, S. 5.

44 Salzborn 2020, ebd. 
Schicksalsnarrative, die sich daraus ergeben, erscheinen als Allheilmittel gegen die Kontingenz moderner Gesellschaften. „Kontingent”, schreibt Niklas Luhmann,

„ist etwas, was weder notwendig ist noch unmöglich ist: was also so, wie es ist (war, sein wird), sein kann, aber anders möglich ist. Der Begriff bezeichnet mithin Gegebenes (Erfahrenes, Erwartetes, Gedachtes, Phantasiertes) im Hinblick auf mögliches Anderssein: er bezeichnet Gegenstände im Horizont möglicher Abwandlungen." 45

Realität wird immer unter der Ägide ihres jederzeit Anders-Sein-Könnens reflektiert. Sie kann verändert werden. Ein Schicksal aber kann nicht geändert werden. Es muss ertragen werden. Genau gegen diese, mit Blumenbergs Wort, "Kontingenzkultur"46 der Moderne wettert die Neue Rechte.

Die zweite Möglichkeit ist, das Ende, das Sieferles Text insinuiert, mit der rhetorischen Figur der Personifikation zusammenzubringen, der sogenannten fictio personae. ,Germania' ist nämlich geradezu ein klassischer Fall für dieses rhetorische Verfahren, das Sieferle sich hier zu eigen macht. Ziel der Personifikation ist es, so lautet die Definition in einem einschlägigen Lexikon, "Unbelebtem (z. B. Naturphänomenen), Abstraktem (Affekten, Tugend/Lastern), Kollektivem (Volk, Land, Kirche) Leben, Bewußtsein und menschliche Gestalt" ${ }^{47} \mathrm{zu}$ verleihen. Sinn der Personifikation ist es also, aus dem, was man nicht sehen kann, etwas Sichtbares zu machen. Übertragen auf den Titel von Sieferles Buch heißt das, dass wir es mit dem Ende des Personifkationsmusters in der politischen Rhetorik zu tun haben.

Die Frau, die Deutschland gewesen ist, nämlich ,Germania', ist verschwunden oder am Ende. Die verbindlichen Bilder, mit und in denen man so etwas Unsichtbares wie das deutsche Volk, sehen' kann, gibt es nicht mehr. Die Zeiten, in denen man mit einem Blick auf Bilder Fragen der nationalen Selbstverständigung klären konnte, sind unwiederbringlich vorbei. Bilder wie Friedrich Overbecks "Italia und Germania", das die deutsche Identität mit ihrem Hang zum Anderen Italiens sehen lässt, ein Gemälde wie Velts "Germania", das 1848 in der Paulskirche hing und den Auf-

45 Luhmann 1993 [1986], S. 152.

46 Blumenberg 1990, S. 57.

47 Huber 2007, S. 53. bruch Deutschlands in eine bessere, weil friedliche Zukunft zum Ausdruck bringt; all diese Bilder sind am Ende, weil nach Meinung Sieferles die Deutschen nicht mehr wissen, wer sie sind oder sein wollen. Das Ende von Germania als Gestalt und als rhetorische Figur firmiert, in durchaus barockem Sinne, als Emblem des Buches.

Die Kritik, die möglicherweise hinter der Rede vom Ende verbindlicher Bilder steckt, ist eine Kritik an den Formen der staatlichen respektive politischen Repräsentation, die im Buch Sieferles als "Herrschaftskultur" (FG, 23) bezeichnet wird. Inkriminiert wird, dass auf die aufwendige und bildhafte Inszenierung der Fiktionen wie Nation, Staat, Freiheit oder gar Volk programmatisch verzichtet wird. Dieses Argument, das ja nahezu ein klassisches gegen die politische Moderne ist, verfängt aber bei Sieferles Kritik an der Bundesrepublik Deutschland nicht. ${ }^{48}$ Der "kleinbürgerliche Charakter der bundesdeutschen Politikszene" (FG, 24) ist ja bekannt. Sie bedeutet aber keine Krise der politischen Repräsentation, sondern fungiert vielmehr als Ausdruck einer veränderten Form von Karriere in der Politik. Der Souverän, das Volk, und seine Macht sind in dieser Form nicht sichtbar. Sie verschwinden im Ikonoklasmus des kleinbürgerlichen Milieus.

Die für die Ikonografie des Politischen fundamentale Unterscheidung zwischen symbolischen und realen Personen funktioniert nicht mehr. Aus diesem Grund können "Konrad Adenauers Wortschatz, Heinrich Lübkes Rhetorik, Ludwig Erhards literarische Bildung, Helmut Schmidts Esssitten, Willy Brandts Trinkgewohnheiten und Helmut Kohls Physiognomie" (FG, 24) zur "leichte[n] Beute" (ebd.) für Parodisten werden.

Der moderne Staat unterzieht sich in der Darstellung dessen, was inn ausmacht, nämlich der Macht, einer ikonischen Askese. Die meisten modernen Staaten haben die Steuerung und Planung ihrer Sichtbarkeit, ihrer Repräsentation in Bildern, Bauten, Kleidern, Souveränitätszeichen, weitgehend aus der Hand gegeben. Repräsentiert werden der Staat und seine Institutionen durch zumeist zeitlich befristet tätige Amtsträger. Sieferles Kritik an der politischen Repräsentation ist eine Reise in die Vormoderne demokratischer Gesellschaften, in denen es, wie Carl Schmitt schreibt, funktionierende bzw. scheiternde Bilder

48 Vgl. Manow 2008. 
oder Gestalten (wie z. B. den sprichwörtlichen ,Leviathan') gegeben habe, in denen man "d[er] Einheit des politischen Gemeinwesens" ${ }^{49}$ ansichtig werden konnte. Diese Bilder sind verschwunden.

Er inkriminiert im Grunde das, was schon Carl Schmitt in seiner Schrift Römischer Katholizismus und politische Form inkriminiert hatte. Politik muss, wenn sie zur Repräsentation fähig sein soll, eine Idee verwirklichen..$^{50}$ Nur so funktioniert dann die politische Kritik, die es ermöglicht, die Differenz zwischen der Idee des Politischen und der Realität politischer Institutionen in den Blick zu nehmen. Sieferles Kritik ist somit nur als zweiter oder gar dritter Aufguss der bekannten Kritik an der politischen Repräsentation zu interpretieren. Er beklagt die vermeintliche Leere der politischen Bilder, in denen wir die Einheit eines oder unseres Gemeinwesens imaginieren.

Ein beliebtes Muster der Gegenwartskritik ist daher die Erfindung einer Vergangenheit, von der sich die Gegenwart radikal unterscheidet. Aber welche Gegenwart hat Sieferle überhaupt im Blick? Weder der Text noch das Nachwort informieren darüber, in welcher Gegenwart sich die Lektüre bewegt. Ist es unsere heutige? Jakob $\mathrm{S}$. Eder hat in der TAZ nachgewiesen, dass es sich bei der Gegenwart, von der Sieferle angeblich spricht, um die Gegenwart der 1990er-Jahre handelt: „Aber Sieferles Ausführungen, die einige Jahrzehnte auf seiner Festplatte Staub angesetzt hatten, nun als Kritik der heutigen Bundesrepublik zu lesen, ist ein ahistorisches Missverständnis." 51 Insgesamt ist der "Geist der neunziger Jahre" 52 , gerade nicht jener Gegenwart im Jahre 2018, prägend für dieses Buch. Der Text Sieferles, der ja aus dem Nachlass stammt und von dem überhaupt nicht klar ist, ob er überhaupt publiziert werden sollte, wird, wenn man so will, in die Gegenwart und in die in ihr drängenden Fragen , migriert'.

Die Vergangenheit, die in der Neuen Rechten ersonnen wird, erscheint so als negatives Gegenbild der Gegenwart. ${ }^{53}$ Sieferle erfindet an diversen Stellen Vergangenheit, z. B. eine Vergangenheit der Landschaft, die er als "Kulturlandschaft"

49 Schmitt 1982 [1938], S. 132.

50 Schmitt 1984 [1923], S. 22.

51 Eder 2017.

52 Eder 2017.

53 Groebner 2008, S. 123-134.
(FG, 58) und als "anthropomorphe[n] Raum" (FG, 58) beschreibt. Eine Kulturlandschaft ist "eine unter konkreten lokalen Bedingungen von menschlichen Aktivitäten geprägte Landschaft, deren Dimensionen auf die Bewegungen und die Arbeit des Menschen bezogen waren" (FG, 58).

Man hört hier fast den älteren Sieferle sprechen. Die Kulturvernichtung durch Industrialisierung kann man, darauf hat Sieferle an anderer Stelle hingewiesen, nur dann beklagen, wenn man Veränderung als Vernichtung und als Bedrohung von Identität wahrnimmt. ${ }^{54}$ Kultur wird hier Ort ursprünglicher Heimat. Genau diese Form von Heimat ist verloren gegangen. Die Übertragungsleistung zwischen Natur, Kultur, Politik und Gesellschaft ist also immer durch sprachliche Bilder konstruiert. Vergleiche, Metaphern und Metonymien ermöglichen so den Übertritt von einem zum anderen, also von der Natur zur Kultur. ${ }^{55}$ Insbesondere sind es die Überlegungen Adam Müllers, die hier einschlägig sind. In seiner berühmten Vorlesung „Elemente der Staatskunst", die Müller 1808 und 1809 gehalten hat, heißt es:

\begin{abstract}
"[D]er Staat ist nicht eine bloße Manufaktur, Meierei, Assekuranzanstalt oder merkantilistische Sozietät: $\mathrm{Er}$ ist die innige Verbindung der gesamten physischen und geistigen Bedürfnisse, des gesamten physischen und geistigen Reichtums, des gesamten inneren und äußeren Lebens einer Nation, zu einem großen energischen, unendlich bewegten und lebendigen Ganzen." ${ }^{56}$
\end{abstract}

Dieses Zitat ist oft verwendet worden, nicht immer zu seinem Besten. Oft übersehen wird, dass die Argumentation, dass der Staat nicht etwa etwas Künstliches, sei, sondern dass sich aus einem bestimmten Verständnis des Menschen und seiner Beziehung zur Natur ableitet.

Diese Frage bearbeitet Müller in der dritten Vorlesung. Dort heißt es: "Die Erde wehrt sich unaufhörlich gegen diese Angriffe ihrer Kinder [Angriffe auf die Natur durch Recht, Eigentum und Politik, MST]." ${ }^{57}$ Er schreibt sogar, dass es einen "Krie[g] des Menschen mit der Erde" 58 gebe, der sich in der Erfindung einer nicht naturgemäßen, sondern künstlichen „Teilung der Arbeit"59 reprä-

54 Sieferle 1997, S. 162ff, Steinmayr 2020.

$55 \mathrm{Vgl}$. Koschorke 2009.

56 Müller 1936 [1808-1808], S. 27.

57 Müller 1936 [1808-1808], S. 37.

58 Müller 1936 [1808-1808], S. 39.

59 Ebd. 
sentiere. Die Vermarktlichung des Gemeinwesens führe zum Verlust von Vielfalt:

\begin{abstract}
„Erst müßt ihr die Erde mit ihren unendlichen Klimaten und eigenthümlichen Lokalitäten in eine große gleichförmige Fläche ausgewalzt haben, erst muß alle Vorliebe der Menschen für das Nähere und Angewöhnte und für das Besondere, Erworbene ausgerottet sein, ehe diese unbedingte Gewerbefreiheit, also ehe dieses absolut freie Privatvermögen der Einzelnen möglich wäre." 60
\end{abstract}

Das Staatswesen darf nicht Ausdruck des ,Krieges' des Menschen gegen die Natur sein, es muss sich gleichsam organisch einfügen in den Kreislauf der Natur. Es ist nicht nur Ausdruck des romantischen Widerstands gegen die Modernisierung von Staat, Recht und Ökonomie. Es ist Ausdruck eines gleichsam, ökologischen' Verständnisses von Staatlichkeit, das die Frage nach der Organisation von Macht anders stellt. Der Bezug auf die Natur ist also eminent politisch.

Der neuere Sieferle überträgt diesen Prozess nun auf die "Beziehungsräume der Menschen" (FG, 59), denen der "Charakter der Persönlichkeit abhanden" (FG, 59) gekommen sei. Diagnose ist "Beziehungsunsicherheit" (FG, 60), die die neue Struktur begründet: nämlich eine solche des "funktionalen, postanthropomorphen Raums" (FG, 60). In einer seltsamen Assonanz an Klages Theorie der mythischen Urbilder schreibt Sieferle: "In den meisten Menschen schlummert noch immer der überkommene anthropo-teleomorphe Zugang zu einer Welt, die sich diesem Zugang immer weiter entzieht" (FG, 60). Ergebnis ist die "Konfrontation zweier Daseinsschichten" (FG, $60)$, die in der Moderne aufeinanderprallen. Die Semantik der Kollision des Vergangenen mit dem Gegenwärtigen zieht sich durch den Text. Sieferle erzählt von Figuren der Anomie und der Unterbrechung. Es entsteht das Bild der Moderne als eines politischen, anthropologischen und historischen Unfalls.

Arbeit an der Geschichte ist immer auch Arbeit an den Begriffen und begrifflichen Oppositionspaaren, mit denen Geschichte narrativ strukturiert werden kann. Für Sieferle ist es die Differenz Politik/System, die er für das begriffliche Oppositionspaar hält, mit dem Gegenwart beschrieben werden kann.

60 Müller 1810 [1921], S. 34.
„Politik", heißt es bei Sieferle,

"gehört einer älteren Daseinsschicht an, geordnet in Hinblick auf Staat und Gesellschaft, kristallisiert in Staatsmännern, Führern und Ideologen. Es gibt in ihr Programme, Werte und Ziele. Gefordert sind Tugenden und Einsätze, die sich auf ein übergeordnetes Ganzes richten. Ultima ratio ist der Krieg; die Bereitschaft zur Selbsthingabe des Individuums für eine höhere Sache, für eine Gemeinschaft, zum Opfertod." (FG, 46f.)

Man sieht ganz deutlich, dass Sieferle hier nicht das moderne Funktionssystem der Politik meinen kann.

Damit gerät ein Politikverständnis in den Blick, das für die von Sieferle selbst untersuchte konservative Revolution entscheidend war. ${ }^{61}$ Was Sieferle hier nämlich als ,Daseinsschicht' beschreibt, ist die societas civilis, jene alteuropäische Referenz des Konservatismus, in der Staat und Gesellschaft geeint sind und die Trennung von oikos/ polis noch intakt gewesen ist. Stärker als mit dem Ausdruck ,Bereitschaft zur Selbsthingabe' kann man die Differenz zur Moderne der bürgerlichen Gesellschaft nicht markieren: Denn in dieser geht es um die Selbsterhaltung und Selbstoptimierung. Sieferle nimmt hier liberalismuskritische Gedanken auf, die für die Traditionsbildung der neuen Rechten entscheidend sind.

Das Gegennarrativ zu diesem alteuropäischen Politikverständnis ist nämlich das System. Mit ,System' beschreibt Sieferle

\begin{abstract}
„Ordnungen höherer Komplexität, welche die Politik sukzessive verdrängen. Systeme organisieren sich ohne Fokus, ohne Werte, Ziele und Programme. Ihre einzige Maxime lautet: Freiheit und Emanzipation für die Individuen. Tugend und Opfer sind Anachronismen, Kriege bloße Konfliktkatastrophen, die es durch geschicktes Management zu verhindern gilt. Ordnung wird durch selbsterzeugte Zwänge der Objektivität geschaffen, nicht aber durch normierende Ausrichtung." (FG, 47)
\end{abstract}

Wenn man genau liest, sieht man, dass dem ,System' eine Eigenschaft fehlt, die in der Sphäre der Politik eine große Rolle spielt: die der Reformierbarkeit. Ein System ist nicht reformier-, nur ersetzbar. Sieferle setzt hier nur wieder einen Diskurs fort, den die konservative Revolution bereits in den Zwanzigerjahren eingeführt hat: Das, was man ablehnt, nämlich das politische

61 Sieferle 1995. 
System der Weimarer Republik sollte nicht reformiert, sondern "ruiniert" 62 werden. So erscheint im Anschluss an das bereits Gesagte das System als Schicksal, dem man nicht entkommen kann.

Mit ,System' beschreibt Sieferle Gesellschaft und Politik der liberalen Moderne, in deren Zentrum der moderne Individualismus steht. Dieses Individuum ist zerfallen in Rollen und Funktionen, die Steuerung der Verhältnisse entzieht sich inm. Atomisierung, Selbstverlust anstatt Selbstopfer, Diskontinuität der Erfahrung, all dies sind bekannte Topoi der modernen Anti-Moderne. Schon Armin Mohler schreibt in seiner Kampfschrift Gegen die Liberalen, in der nicht die Linke, sondern der Liberalismus als Hauptgegner im Politischen ausgemacht wird:

"[D]as Individuum gibt es gar nicht. Es ist eine Erfindung. Die Vorstellung eines autonomen Individuums, wie sie dem Liberalen so am Herzen liegt, ist die schlimmste aller Abstraktionen. Es ist geradezu banal, das festzustellen: jeder Mensch steht in einem Lebenszusammenhang, von dem aus er denkt und reagiert." ${ }^{63}$

Sieferle inszeniert sein Schreiben als meditative Fundamentalopposition zur gesellschaftlichen Modernisierung. Diese Modernisierung ist für ihn gleichsam das Fatum. Ihm ist nicht zu entrinnen. Wilhelm Windelband hatte bereits 1878 das Skript dazu geliefert. In seinem Hölderlin-Vortrag heißt es:

\footnotetext{
„Die Kultur ist zu breit geworden, um vom Standpunkt des Individuum aus übersehen zu werden [...] Das Bewußtsein des einheitlichen Zusammenhanges, der alles Kulturleben beherrschen soll, geht Schritt für Schritt verloren und die Gesellschaft droht in Gruppen und Atome zu zerfallen, zwischen denen es nicht mehr das Bindemittel des geistigen Verständnisses, sondern nur noch dasjenige der äußeren Not und Notwendigkeit gibt. So wird die moderne Gesellschaft mehr und mehr zu einem Bild der Zerrissenheit, und je schneller dieser Prozeß mit natürlicher Notwendigkeit fortschreitet, um so geringer wird selbstverständlich die Kraft der gesellschaftlichen Ordnung, deren festeste Stütze die Gleichheit des Kulturbewußtseins in den Individuen bildet." 64
}

62 Ottmann 2010, Bd. 4/1, S. 144.

63 Mohler 2010, S. 14.

64 Windelband, Wilhelm: Über Friedrich Hölderlin und sein Geschick (1878). In: W. W.: Präludien I, 9. Aufl. Tübingen 1924, S. 254f. Zitiert in: Breuer 1993, S. 20.
Windelband beschreibt in seiner Rede den „völligen Zusammenbruch" 65 der vertrauten bildungsbürgerlichen Welt. Was Windelband und Sieferle miteinander verbindet, ist die vermeintliche Erfahrung kultureller Enteignung. Das Interessante an der Rhetorik der Neuen Rechten ist aber, dass sie nicht nur der bildungsbürgerlichen Klasse Eigentumsrechte zuschreibt, sondern einem Volk. Die Strategie ist also eine der Übertragung von althergebrachten Narrativen und Symbolstrukturen: Aus den historischen Verlusten an Repräsentation, Leser*innenschaft, Geschichte, Identität und Verbindlichkeit wird das Recht eines Volkes auf Aneignung eben dieses vermeintlich ,Entwendeten'. Es geht um Restitution des Gewesenen, nicht um Reform des Gegenwärtigen. Dieses Narrativ, das die Neue Rechte als ihr Ureigenes feiert, entpuppt sich bei genauer Betrachtung als eine Wiederholung, als geistes- und sozialgeschichtliche Redundanz. Das ,Neue' an der Neuen Rechten erweist sich als geistesgeschichtliche Diskurspatina. Somit ist Sieferles Text nicht anderes als das Lamento einer ehemals bürgerlichen Klasse, die sich mit dem Volk verwechselt. Das Lamento kommt als politischer Schwulst daher.

\section{Björn Höcke: Nie zweimal in denselben Fluss}

Nimmt man Björn Höckes Nie zweimal in denselben Fluss zur Hand, so fällt zunächst die Gestaltung des Umschlags auf. Der/die Leser*in sieht ein Rednerpult mit einem Mikrofonkranz. Das nicht gleichschenklige Dreieck beginnt am linken Rand des Buchrückens und zieht sich über die Frontseite, um dann außerhalb des Buches zu enden. Die strengen geometrischen Formen lehnen sich an die faschistische Formensprache an. Bevor der/die Leser*in das Buch aufschlägt, um zu lesen, ist seine Phantasie mit einem Sprechenden besetzt. Dazu passt auch der Reihentitel: „Politische Bühne. Originalton", der sich fett gedruckt auf dem Broschurumschlag findet.

Der hier in Szene gesetzte Gegensatz liegt auf der Hand: Ein Buch, das man lesen soll, wird durch die Rolle des Protagonisten als Redner im öffentlichen Raum verstärkt oder ergänzt. Der Verlag

65 Breuer 1993, S. 20. 
scheint zu wissen, was schon Adolf Hitler gewusst hat. Im Vorwort von Mein Kampf heißt es:

"Ich weiß, daß man Menschen weniger durch das geschriebene Wort als vielmehr durch das gesprochene Wort zu gewinnen vermag, daß jede große Bewegung auf dieser Erde ihren Wachsen den großen Rednern und nicht den großen Schreibern verdankt."66

Björn Höcke ist nämlich bisher nicht als, großer Schreiber' hervorgetreten. Vielmehr ist seine Rolle in der AfD die des Redners. In diesem Medium kann er die Menschen, gewinnen'. Welchen Zweck hat also die Verschriftlichung? Zunächst muss man sehen, dass es sich um einen Interviewband handelt. Björn Höcke tritt hier wieder als Sprecher in Erscheinung, dem es durch Sebastian Hennig ermöglicht wird, im Medium des gesprochenen Wortes zu reüssieren. Es wird eine orale Gemeinschaft adressiert. Oralität bezeichnet hier den Status einer Kultur, in der die Kommunikation und das sie tragende Medium des gesprochenen Wortes Tradition speichert und weitergibt. Die Worte sind in einer oralen Kultur keine Zeichen, sondern, wie Walter J. Ong wusste, "Klänge, [...] sie hinterlassen keine Spur, sie sind Erscheinungen, Ereignisse". ${ }^{67}$ Die Wissensübermittlung verläuft über das Gespräch oder die gehörte Rede, das oder die bestimmten mnemotechnischen Mustern folgt, um die Weitergabe des Wissens zu gewährleisten: Iteration, Alliteration und Assonanz, Epitetha sind mithin die bevorzugten Mittel, um die Memorierbarkeit von gesprochener Sprache zu gewährleisten. Wesentliche Merkmale eines der Oralität verpflichteten Denkens sind, so Ong, ein eher auf konkrete Situationen sich kaprizierendes als nach abstrakten Theoremen verfahrendes Denken. ${ }^{68}$ Die AfD ist also eine Partei der Oralität. Der emphatische Bezug auf das gesprochene Wort und die Macht der Rede, der in der AfD und speziell bei Höcke notorisch ist, hat eine lange Tradition im rechten Denken, in die Höcke und die AfD sich einschreiben.

Aus diesem Grunde heißt es auch in Mein Kampf, dass die "größten Umwälzungen auf dieser Welt [...] nie durch einen Gänsekiel geleitet worden" 69 seien. "Die Macht aber", heißt es wei-

66 Hitler 2016 [1924], S. 89.

67 Ong 1987, S. 37.

68 Ong 1987, S. 42-61.

69 Hitler 2016 [1924], S. 327. ter, "die die großen historischen Lawinen religiöser und politischer Art ins Rollen brachte, war seit urewig nur die Zauberkraft des gesprochenen Wortes". ${ }^{70}$ Der Antiintellektualismus lässt sich also jetzt genauer einordnen. Er erscheint als Opposition gegen die Buch- und Textwelten der Intellektuellen, gegen den Dialog der Meinungen, der in ihnen ausgefochten wird. Bekenntnisse und Authentizitätswahn ersetzen Argumente. Die verlorene Nahwelt der oralen Gemeinschaft wird zum neurechten Kommunikationsideal.

Die Andeutungen zur Medienstrategie in Höckes Buch führen unmittelbar zu einer anderen gewichtigen Frage. Wie kann man das Buch gattungsmäßig einordnen? Das Inhaltsverzeichnis umfasst sechs Abschnitte. Begonnen wird mit "Frühe Jahre", dann geht es über zu "Im Schuldienst", worauf der Abschnitt "Der Weg in die Politik" folgt, um daran anschließend "Partei und Fraktion in Thüringen" in den Blick zu nehmen. Abschließend folgen zwei Kapitel, die nicht zwingend dem Lebensweg folgen, sondern mit den Titeln "Volksopposition gegen das Establishment" und "Krise und Renovation" sich vom Lebensbericht trennen. ${ }^{71}$ Der Hauptteil des Buches ist somit als autobiografisch anzusehen, wobei die Erzählung vom eigenen Leben mit dem Eintritt in die Politik endet.

Das Buch übernimmt hier teilweise den Aufbau von Hitlers Mein Kampf. ${ }^{72}$ Die eigentlichen autobiografischen Kapitel in Hitlers einzigem Buch sind die Kapitel "Im Elternhaus" und "Wiener Lehr- und Leidensjahre", ferner sind in "Allgemeine politische Betrachtungen aus meiner Wiener Zeit", "Sonstiges" und in anderen Abschnitten autobiografische Splitter zu erkennen, die aber dann mit der Geschichte der nationalsozialistischen Bewegung verwoben werden. ${ }^{73}$ Wichtig ist hier das Schema des autobiografischen Umschlags, der in die Politik führt.

Bei Höcke, ähnlich wie bei Hitler, handelt es sich, um hier Annie Ernaux' Begriff zu verwenden,

70 Ebd.

71 Höcke 2020. Hinfort wird Höckes Buch mit der Sigle $\mathrm{H}+$ Seitenzahl zitiert.

72 In der bisherigen Rezeption des Höcke'schen Textes sind die Parallelen zu Hitlers Mein Kampf bisher nur ansatzweise thematisiert worden. Vgl. Bittner 2017.

$73 \mathrm{Vgl}$. hierzu die Einleitung der Herausgeber von Hitlers Mein Kampf, Abschnitt "Entwurf einer Lebensgeschichte", S. 30-34. 
um "Autosoziobiografien"74. Autosoziobiografien unterscheiden sich von Selbsterlebensbeschreibungen durch eine Vermischung von Gesellschaftsanalyse mit der Arbeit am Selbst. Sie lassen sich als Narrativierung von sozialen Prozessen und ihrer Interpretation, wie etwa Bildungsaufstieg oder -abstieg, Logik der sozialen Reproduktion bzw. Widerstand gegen diese, beschreiben. Autosoziobiografien sind also Selbst- und Zeitdiagnostik in einem. Insbesondere an der Inszenierung von Bildungserlebnissen bzw. vom Scheitern in etablierten Bildungsinstitutionen lässt sich das Funktionieren von Autosoziobiografien zeigen.

Gleichzeitig offenbart sich in der Autosoziobiografie auch eine Politik der Lebensgeschichte. ${ }^{75}$ Eine Politik der Lebensgeschichte organisiert Oppositionen und Schematismen, die das autobiografische Subjekt als Figur überhaupt erst im Diskurs relevant werden lassen. Eine Opposition, die Adorno für die neurechte Politik der Lebensgeschichte vorgeschlagen hat, ist die zwischen Provinz und Land. Adorno sagt in seinem jüngst editierten Vortrag "Aspekte des neuen Rechtsradikalismus", dass es in "diesen [den rechtsradikalen Bewegungen, MST] insgesamt so etwas wie einen sich verschärfenden Gegensatz der Provinz gegen die Stadt"76 gebe. Höcke macht aus dieser Opposition ein Programm, das er auch noch pädagogisch verklärt: „Es gibt pädagogisch nichts Wertvolleres, als das Aufwachsen auf dem freien Land, mit Tieren, mit Abenteuern und der Möglichkeit, unter überschaubaren eigenen Grenzen zu lernen." $(H$, 33.) Das klingt nach pädagogischer Lust auf gesellschaftliche Einfachheit. Aber, spricht Höcke zu Hennig, "[w]enn Sie sich die Biographien der deutschen Dichter und Denker anschauen, werden Sie feststellen, daß nahezu alle auf dem Land oder in den kleinen Ortschaften aufwuchsen, auch wenn später ihre Namen mit großen Städten verbunden wurden." $(H, 33$.$) Die$ Provinz sorgt für die Herkunft, ja für Heimat und ein Heimatgefühl, der gesellschaftliche Aufstieg aber findet in den Städten oder in der Metropole statt. Aber eine Identität stiften die Städte nicht.

Höckes Begeisterung für die heimatliche Scholle ist die Wiederkehr der Heimatkunst von

74 Spoerhase 2017, S. 27-37.

75 Vgl. Schneider 1999.

76 Adorno 2019 [1968], S. 15.
1900 um 2018. Der "anti-städtische Affekt"77 dieser Literatur besteht gerade in ihrer Opposition zur Stadt. Die Stadt gilt als Ort, als Raum der Vergesellschaftung, in dem es unmöglich ist, eine Identität jenseits der gesellschaftlichen Rolle auszubilden. Wenn Julius Langbehn schreibt, dass der "Gelehrte seinem Wesen nach international, der Künstler national"78 sei, dann wird auch der Antiintellektualismus als politische Strategie deutlich. Der "Geist der Individualität", heißt es bei Langbehn, ist der "Geist der Scholle". ${ }^{79}$ Nur auf dieser kann der Deutsche sich verwirklichen und eine Identität ausbilden. International sind immer nur die anderen.

Die verschütteten "kulturellen Quellen" $(H, 30)$ der deutschen Identität, wie Dichtung, Preußen usw., sollen wieder sprudeln. Deutschland ist für Höcke vor allem Natur und ein Netz aus Autorennamen, die so etwas wie die Walhalla der Neurechten bilden. In der Mitte sitzen Nietzsche $(H, 57)$, Heidegger $(H, 59$ und passim), Kant $(H, 60)$, die "deutsche Mystik" $(H, 72)$, Adelbert von Chamisso $(H, 131)$ Martin Buber $(H, 83 f$.) und einige andere Geistesgrößen wie Edmund Burke $(H, 70)$, Macchiavelli $(H, 153$ und passim). Im Verlauf des Buches entspinnt sich für den/die Leser*in ein Netzwerk von alt- und neurechten Autoren: Wolfgang Caspart $(\mathrm{H}, 105)$, Joachim Fernau ( $H$, 112), Arnold Gehlen $(H, 128)$, Jordis von Lohausen $(H, 182)$, Manfred Kleine-Hartlage $(H, 186)$ und, natürlich, Spengler $(H, 263)$. Von Sebastian Hennig kommt der unvermeidliche Verweis auf die konservative politische Romantik mit der Nennung von Achim von Armins Die Kronenwächter und Bettina von Arnims Dies Buch gehört dem König $(\mathrm{H}, 157)$.

Übernimmt man hier Hugo von Hofmannsthals Ausdruck, so entsteht das Deutschland der Neurechten aus Schriften, die den geistigen Raum dessen bilden, was die Neurechte in ihren Schriften als Nation erfindet. Das von Hofmannsthal so genannte "geistig[e] Anhangen" 80 bezieht sich aber nicht auf die großen Werke, sondern, wie Hofmannsthal schreibt, auf

77 Rossbacher 2000, S. 304.

78 Langbehn 1890, S. 9.

79 Langbehn 1890, S. 40.

80 Hofmannsthal 1955 [1927], S. 390. 
"Aufzeichnungen aller Art, wie sie zwischen den Menschen hin und her gehen, den nur für einen oder wenige bestimmten Brief, die Denkschrift, desgleichen auch die Anekdote, das Schlagwort, das politische oder geistige Glaubensbekenntnis, wie es das Zeitungsblatt bringt, lauter Formen, die ja zuzeiten sehr wirksam werden können." 81

Die oben genannten Autoren, die jeweils ganz unterschiedliche Textsorten produziert haben, haben gemeinsam, dass sie in neurechten Kreisen offenbar alleine durch die Tatsache, dass sie gelesen werden, eine Wirksamkeit entfalten. Für den/die Leser*in entsteht so etwas wie ein Kanon neurechten Denkens, der manche Überraschung aufweist und an dem in anderen Publikationen weitergearbeitet wird. Es wird inszeniert, was Umberto Eco "Kult der Überlieferung"82 genannt und als Merkmal des Faschismus identifiziert hat. Es geht also um den Kult, nicht um die Überlieferung an sich.

Der Kanon bekommt unter Rechten Funktion. Das entstandene "Corpus von Texten, das eine Gesellschaft oder eine Gruppe für wertvoll hält und an dessen Überlieferung sie interessiert ist" ${ }^{83}$ wird enthistorisiert. Der Kanon stellt also immer einen Selektionsprozess dar, dessen Ziel darin besteht, einen alternativen oder anderen Überlieferungsprozess zu initiieren. Genau um diesen Überlieferungsprozess geht es hier. In diesem Prozess fungiert der Kanon als „Prägewerk der Identität" ${ }^{\prime \prime 4}$, dessen Ergebnis unterschiedlich sein muss. Ein Kanon ist weder zeit- noch geschichtslos, er kann nicht, wie die neuen und alten Rechten es tun, in einer kultischen Verehrung überliefert werden.

All diese Strategien sind also bekannt. Die neue Rechte liest gerne und viel, erfindet einen Kanon zur kulturellen Selbstverständigung. Was aber bisher in der Rezeption untergegangen ist, ist die Frage nach der Inszenierung von Bildungsfiguren in der Literatur der nationalsozialistischen und neuen Rechten. Mit dieser Frage ist ein wesentlicher Punkt in der Selbstinszenierung genannt: die Inszenierung von Bildung jenseits von Institutionen oder von institutioneller Kommunikation. Bildung außerhalb der Kontrolle durch institutionelle Curricula und Wissenstechni-

81 Hofmannsthal 1955 [1927], S. $390 f$.

82 Eco 2020, S. 11.

83 Winko 1996, S. 585.

84 Assmann 1988, S. 59. ken ist ,halbe Bildung', weil ihr die institutionelle Kontrolle oder die gesellschaftliche Resonanz bzw. Anerkennung fehlt.

Adorno hat den Halbgebildeten „Drang nach Höherem" 85 attestiert und "Verwirrung und Obskurantismus" 86 im Denken als Kennzeichen von Halbbildung destilliert. Von Adornos Theorie der Halbbildung zur Bildungsfigur des Autodidakten ist es nicht weit. "Der Autodidakt", heißt es bei Georg Stanitzek, "hat zwar an Studium und Produktion des Wissens teil, aber nicht, oder wenigstens nicht im Vollsinn, an dessen sozialer Organisation." ${ }^{87}$ Man muss sich den Autodidakten als einsamen Leser vorstellen, der sich idiosynkratisch eine Textwelt zusammenbastelt, die nicht durch eine Organisation kontrolliert wird. Genau in diesem Jenseits der Institutionen scheint er für die Neue Rechte interessant zu sein. „Heute”, gesteht Höcke zunächst, komme ihm das zwar recht abenteuerlich vor, aber

\begin{abstract}
„ich hatte mir in den Kopf gesetzt, Sein und Zeit zu lesen ohne jede Sekundärliteratur und jede Anleitung durch einen Lehrer. Ich glaube, das schwierige Unterfangen hat sich gelohnt, auch wenn es mir schwerfällt, konkrete Früchte vorzuweisen. Schon der Mut Heideggers, das Sein gegenüber dem Seienden ins Recht zu setzen, ist ein Erlebnis." ( $H, 77$, Kursivierung im Original)
\end{abstract}

Dieses Heidegger-Erlebnis, das ja kein Erlebnis einer Analyse ist, sondern ein 'Erleben der ontologischen Differenz, verbindet inn von der Struktur her mit den zahlreichen Autodidakten der Bildungsgeschichte. Denn diese sind, so heißt es bereits 1835, "sich selbst Bildende". ${ }^{88}$ Im weiteren Verlauf der entwickelten Beschreibung des Autodidakten spricht Wolff davon, dass "[g]ute Exempel und zufällige gute Bücher allerdings oft mehr, als Lehrer [bilden]". ${ }^{89}$ Bereits Armin Mohler attestiert sich "maßlos[e] Lesewut"90 jenseits der etablierten disziplinären Wege. Die Dissidenz des Bildungszugangs und die Ablehnung von wissenschaftlicher Kommunikation ist für das Verständ-

85 Adorno 1998 [1962], S. 112.

86 ebd.

87 Stanitzek 1999, S. 327.

88 Wolf 1835, S. 14.

89 Wolf 1835, S. 14.

90 Mohler, Armin: Der Nasenring. Vergangenheitsbewältigung vor und nach dem Fall der Mauer. München 1991, S. 32ff. Zitiert nach: Maas 2013, S. 43. 
nis und für die Tradition des neurechten Erzählsystems von erheblicher Bedeutung.

„Die Fachwissenschaft", heißt es dementsprechend in Goebbels' einzigem Roman Michael. Ein deutsches Schicksal in Tagebuchblättern, „züchtet Hochmut und Fachsimpelei". ${ }^{91}$ Aus diesem Grunde sind die Lektüren des anfänglich noch studierenden Michael einsam. Er liest Goethes Wilhelm Meister, den er nicht als Roman, sondern als "Epos"92 wahrnimmt. Beim Besuch in Frankfurt, wo Michael anscheinend das GoetheHaus besucht hat, heißt es zum Bild der Charlotte Buff, die ja bekanntlich das Vorbild für Lotte in Die Leiden des jungen Werthers gewesen ist: "Das war noch der Goethe, wie wir Jungen inn liebten. Der Geheimrat ist manchmal unausstehlich." ${ }^{93}$ Er liest auf Vermittlung seines russischen Freundes Dostojewskis Der Idiot und bezeichnet Fürst Myschkin als "haltose[n], unberechbare[n], lächerliche[n] Menschen". ${ }^{94}$ Er sitzt in der Natur und liest Kellers Der grüne Heinrich, jenes, wie er in eigentümlicher Diktion formuliert, "Buch von der dämmernden Ferne und der festen männlichen Gegenwart". ${ }^{95}$ Der kriselnde Student liest die Fröhliche Wissenschaft. ${ }^{96}$

Der bürgerliche Kanon wird umgedeutet, neu besetzt und für die eigenen Zwecke hergerichtet. Die "Weitergabe deutscher und europäischer Kulturtraditionen" $(H, 96)$, die Höcke ins Zentrum seiner "Bildungsbemühungen" $(H, 96)$ stellt, ist keine. Es ist eine Umbesetzung von Kulturtraditionen, in der genau diese Tradition neu erfunden wird. Es scheint, als imitiere Höcke mit diesem Verfahren Oswald Spengler, der ja im Rahmen seiner Morphologie der Geschichte permanent politische und künstlerische Formen der Antike, des Mittelalters, der Frühen Neuzeit und der Moderne zueinander in Beziehung setzt. Diese Beziehungslehre definiert den literarischen Stil. Im Abschnitt "Philosophie der Politik" heißt es exemplarisch für diesen Stil bei Spengler:

\footnotetext{
91 Goebbels, Joseph 1942 [1929], S. 14.

92 Goebbels 1942 [1929], S. 17.

93 Goebbels 1942 [1929], S. 18.

94 Goebbels 1942 [1929], S. 33.

95 Goebbels 1942 [1929], S. 43.

96 Goebbels 1942 [1929], S. 51.
}

„Aber während die Antike, an der Spitze das Forum von Rom, die Volksmasse zu einem sichtbaren und dichten Körper zusammenzog, um ihn zu zwingen, von seinen Rechten den Gebrauch zu machen, den man wollte, schuf, gleichzeitig' die europäisch-amerikanische Politik durch die Presse ein Kraftfeld von geistigen und Geldspannungen über die ganze Erde hin, in das jeder einzelne eingeordnet ist, ohne daß es ihm zum Bewußtsein kommt, so daß er denken, wollen und handeln muß, wie es irgendwo in der Ferne eine herrschende Persönlichkeit für zweckmäßig hält." ${ }^{17}$

Auf die Frage von Sebastian Hennig, ob Höcke sich als Populist bezeichnen würde, antwortet dieser: "Richtig liegt er [der Populismus, MST] in der Diagnose, daß sich im Zuge der Oligarchisierungsund Ochlokratisisierungstendenzen eine abgehobene polit-mediale Kaste gebildet und vom Volk entfremdet hat." $(H, 235$.$) Neben dem Verweis$ auf Polybios, dessen Geschichtsschreibung Höcke vorher anzitiert hat $(H, 225)$, zitiert Höcke zumindest implizit Goebbels' Michael: „Ach heute! Das ist ja keine Politik, was die da oben betreiben. Sie führen nur mit den Mitteln des Volkes [lic. Steuergelder, MST] ihr eigenes Geschäft. Unsere sogenannte Politik steht in keinem inneren Verhältnis mehr zum Volk." 98 Höcke inkriminiert im Lichte dieses Zitats die staatliche Parteienfinanzierung, dementsprechend als "Wirtschaftszweig" $(H, 58)$, er sieht Intellektuelle, die "an Universitäten und Hochschulen ungeniert Steuergelder abgrasen" $(\mathrm{H}, 180)$.

Ich habe meinen Durchgang durch neurechte Textwelten mit einem Blick auf den modernisierungsskeptischen Goethe begonnen. Die neue Rechte versucht, eine andere Moderne zu erfinden. Sie versucht nicht, sie zu verstehen. Ihr Ansinnen ist es, so kann man sagen, ihre Sicht auf die gesellschaftliche Moderne mit dem Blick auf ihre kulturell-ästhetische Moderne zu versöhnen. Der Kanon von Langbehn, Hitler, Schmitt bis hin zu Heidegger ist der Spiegel dieses Versuchs. Die Idee der Einheit, der Vorstellung sozialer und politischer Homogenität, die Phantasie einer Identität zwischen Sein und Zeichen lassen sich im Anschluss an die Analysen Nassehis nicht nur als soziologische, sondern auch als ästhetischkulturelle Strategien beschreiben.

Wie Ingo Stöckmanns Untersuchungen zur Genese und zur Problematik der literaturwissenschaftlichen Moderne ${ }^{99}$ gezeigt haben, ist das,

97 Spengler 1998 [1923], S. 1137.

98 Goebbels 1942 [1929], S. 21.

99 Stöckmann 2009, 2012. 
was Moderneerfahrung in der Literatur sein kann, auf Narrativierung der Erfahrung und auf Organisation von Narrativen konstitutiv angewiesen. Moderne, so kann man sagen, ist für ihre literarisch-kulturelle Verarbeitung auf Erzählbarkeit, ja, auf Transformation in erzähl- oder imaginierbare Elemente angewiesen. Im Ergebnis wird moderne Literatur dann Bilanz jener Moderne, die man als Verlustrechnung oder als Gewinnbuchung erzählen kann.

Die Funktion der "Welterschließung und Daseinsaufschlüsselung"100, die Kubitschek und Kositza daher dem Lesen zusprechen, ist eine Erfahrung der Moderne in der Literatur und im Lesen. Zu dieser Moderne bzw. zur liberalen Moderne gehört die Auseinandersetzung mit dem Anderen der eigenen Meinung. Schon Adorno hat die Verweigerung der Diskussion als Element des Rechtsradikalismus analysiert: "So wurde neulich in einer Polemik gegen eine Professorin, die den Rechtsradikalen nicht in den Kram paßte, gesagt: ,Wir diskutieren nicht mit ihr, sondern hier handelt es sich um existentielle Gegensätze'."101 Denn Auseinandersetzung oder Diskussion sind etwas anderes als der Verweis auf Existentielles, Rückzug in Lektüregemeinschaften, politische Neosemantik, Wälder oder Schlösser.

In Eichmann in Jerusalem referiert Hannah Arendt auf das Schlusswort des Angeklagten Eichmann, in dem dieser von der "staatlicherseits vorgeschriebenen Umwertung aller Werte gesprochen"102 habe. Die Umwertung aller Werte der Tradition ist ja etwas anderes als sie aufzugeben. Die neue Rechte will diesen umwertenden Staat. Sie bereitet inn vor. Das berühmte Arendt'sche Wort von der "Gedankenlosigkeit"103 des HolocaustKoordinators wird ergänzt durch die attestierte "Realitätsferne"104 Eichmanns. Es ist die unselige Kombination aus Gedankenlosigkeit, Armut an politischer Phantasie und Realitätsferne, die nach Arendt "mehr Unheil anrichten können als alle die in einem Menschen innewohnenden bösen Triebe zusammengenommen". ${ }^{105}$ Die beiden hier untersuchten Autoren sind sicherlich keine Eichmän-

100 Kositza/Kubitschek 2020, S. 8.

101 Adorno, Aspekte des neuen Rechtsradikalismus, S. 34.

102 Arendt 1986 [1961], S. 57.

103 Ebd.

104 Ebd.

105 Ebd. ner. Und doch: Die Banalität der Aussagen und den Eklektizismus ihrer Texte kann man als Ausdruck von Gedankenlosigkeit und Realitätsferne lesen. Sie offenbaren eine politische arme, kulturell dürftige und literarisch armselige Phantasie und Einbildungskraft. Es ist alles schon einmal dagewesen.

\section{Literatur}

Adorno, Theodor W. (2019): Aspekte des neuen Rechtsradikalismus. Ein Vortrag [1968]. Mit einem Nachwort von Volker Weiß. Berlin: Suhrkamp.

Adorno, Theodor W. (1998): Theorie der Halbbildung [1962]. In: ders.: Gesammelte Schriften in zwanzig Bänden. Darmstadt: WBG [=Lizenzausgabe STW, Frankfurt 1997], Bd. 8, S. 93-122.

Arendt, Hannah (1986): Eichmann in Jerusalem. Ein Bericht von der Banalität des Bösen [1961]. München: Piper.

Aristoteles (1982): Poetik. Griechisch/Deutsch. Übersetzt und herausgegeben von Manfred Fuhrmann. Stuttgart: Reclam.

Assmann, Aleida (1998): Kanonforschung als Provokation der Literaturwissenschaft. In: von Heydebrand, Renate (Hg.): Kanon-Macht-Kultur. Theoretische, historische und soziale Aspekte ästhetischer Kanonbildungen. Stuttgart: J. B. Metzler, S. 47-59.

Beyer, Susanne (2017): Menschenwerk. Die Empfehlung einer Essaysammlung mit rechtsradikalen Inhalten durch einen SPIEGEL-Redakteur bedarf einer Erklärung. Reaktion der Spiegel-Redaktion. URL: https://www.spiegel.de/spiegel/print/d-151666500. html (28.04.21).

Bittner, Michael (2019): Höcke und sein Kampf, 03.09.2021, URL: https://michaelbittner. info/2019/09/03/bjoern-hoecke-und-sein-kampf/ (17.09.2020).

Blumenberg, Hans (1990): Die Sorge geht über den Fluß. Frankfurt/M.: Suhrkamp.

Breuer, Stefan (1993): Anatomie der konservativen Revolution. Darmstadt: WBG.

Czollek, Max 2020: Desintegriert Euch! [2018] München: BTB.

Detering, Heinrich (2019): Was heißt hier, wir'? Zur Rhetorik der parlamentarischen Rechten. Stuttgart: Reclam.

Dobrenko, Evjenij (2011): Stalins Schreibweise. Von den romantischen Dichtungen der Zukunft zur sozialistisch-realistischen Prosa der Vergangenheit. In: Koschorke, Albrecht/Kaminskij, Konstantin (Hg.): Despoten dichten. Sprachkunst und Gewalt. Konstanz: UVK, S. 97-177.

Eco, Umberto (2020): Der ewige Faschismus. Aus dem Italienischen von Burkhard Kroeber. München: Hanser.

Eder, Jakob S. (2017): Zurück in die Diskursvergangenheit. Rechte Mythen im Buch „Finis Germania”. 
URL: https://taz.de/Rechte-Mythen-im-Buch-FinisGermania/!5441743/ (28.04.21).

Felsch, Philipp (2015): Der lange Sommer der Theorie. Geschichte einer Revolte 1960-1990. München: Beck.

Fücks, Ralf/Becker, Christoph (Hg.) (2020): Das alte Denken der Neuen Rechten. Die langen Linien der antiliberalen Revolte. Frankfurt/M.: Wochenschau.

Gess, Nicola (2021): Halbwahrheiten. Zur Manipulation von Wirklichkeit. Berlin: Matthes \& Seitz.

Goebbels, Joseph (1942): Michael. Ein deutsches Schicksal in Tagebuchblättern [1929]. München: Franz-Eher.

Goethe, Johann Wolfgang von (2005): Maximen und Reflexionen. Aus dem Nachlaß (Nr. 876). In: ders.: Sämtliche Werke nach Epochen seines Schaffens. Münchner Ausgabe. Hg. v. Karl Richter in Zusammenarbeit mit Herbert G. Göpfert, Norbert Miller, Gerhard Sauder u.a. 21 Bde. München: btb, Bd. 17, S. 715-957.

Graevenitz, Gerhart von (1973): Die Setzung des Subjekts. Untersuchungen zur Romantheorie. Tübingen: Niemeyer.

Groebner, Valentin (2008): Das Mittelalter hört nicht auf. Über historisches Erzählen. München: Beck.

Grossarth, Jan (2017): Redakteur des "Spiegel" gab rechtsextreme Leseempfehlung. URL: https://www.faz. net/aktuell/politik/inland/wer-setzte-rechtsextremesfinis-germania-auf-ndr-buchliste-15055693/ das-umstrittene-buch-finis-15058149.html. (28.04.21).

Hitler, Adolf (2017): Mein Kampf [1924]. Eine kritische Edition, hg. v. Christian Hartmann, Thomas Vordermayer u. a. Im Auftrag des Instituts für Zeitgeschichte. München-Berlin: Institut für Zeitgeschichte.

Höcke, Björn (2020): Nie zweimal in denselben Fluss. Björn Höcke im Gespräch mit Sebastian Hennig. Mit einem Vorwort von Frank Böckelmann. 4. Aufl. Lüdinghausen und Berlin: Manuscriptum.

Hofmannsthal, Hugo von (1955): Das Schrifttum als geistiger Raum der Nation [1927|. In: ders.: Gesammelte Werke in Einzelausgaben: Prosa IV, Frankfurt/Main 1955, S. 390-414.

Huber, Christoph (2007): Personifikation. In: Reallexikon der deutschen Literaturwissenschaft, Neubearbeitung des Reallexikons der deutschen Literaturgeschichte, hrsg. v. Klaus Weimar, Bd. 3: P-Z, Berlin u.a.: De Gruyter, S. 53-55.

Kellerhoff, Sven Felix (2015): "Mein Kampf". Die Karriere eines deutschen Buches. Stuttgart: Klett-Cotta.

Kiesel, Helmut (2014): War Adolf Hitler ein guter Schriftsteller? In: Frankfurter Allgemeine Zeitung, 4.08.2014, Nr. 178, S. 11.

Koch, Lars (Hg.) (2020): Zwischen Feindsetzung und Selbstviktimisierung : Gefühlspolitik und Ästhetik populistischer Kommunikation. Frankfurt/M.: Campus.

Koschorke, Albrecht (2009): Die Epistemologie der Natur/ Kultur-Grenze und ihre disziplinären Folgen. In: Deutsche Vierteljahresschrift für Literaturwissenschaft und Geistesgeschichte (DVjs) 81/1, S. 9-25.
Koschorke, Albrecht (2016): Adolf Hitlers, Mein Kampf. Zur Poetik des Nationalsozialismus. Berlin: Matthes \& Seitz.

Kositza, Ellen/Sommerfeld-Lethen, Caroline (2019): Vorlesen. Schnellroda: Antaios.

Kositza, Ellen/Kubitschek, Götz (2020): Das Buch im Haus nebenan. Schnellroda: Antaios.

Kubitschek, Götz (2017): Provokation. Schnellroda: Antaios.

Langbehn, Julius (1890): Rembrandt als Erzieher. Von einem Deutschen. 16. Aufl. Leipzig: von Hirschfeld.

Lehmann, Johannes F. (2019): Wut Wut, Zorn, Hass - zum affektpolitischen Problem der Identität. Manuskript d. Verfassers, 81 Seiten.

Leo, Per/Steinbeis, Maximilian/Zorn, Daniel-Pascal (2017): Mit Rechten reden. Ein Leitfaden. Stuttgart: Klett-Cotta.

Ludwig, Cornelius (2017): Genre. In: Metzler Lexikon Literatur. Begriffe und Definitionen. 3. völlig neue bearbeitete Auflage. Hg. v. Dieter Burdorf u. a. Stuttgart-Weimar: J. B. Metzler.

Luhmann, Niklas (1993): Soziale Systeme. Grundriß einer allgemeine Theorie [1984]. Frankfurt/M.: Suhrkamp.

Maas, Sebastian (2017): Die Geschichte der neuen Rechten in der Bundesrepublik Deutschland. Kiel: Regin.

Manow, Philipp (2008): Im Schatten des Königs. Die politische Anatomie demokratischer Repräsentation. Frankfurt/M.: Suhrkamp.

Mohler, Armin (2010): Gegen die Liberalen. Schnellroda: Antaios 2010.

Müller, Adam (1921): Von der Gewerbefreiheit [1810]. In: ders.: Ausgewäh/te Abhandlungen. Hg. v. J. Baxa. Jena: Fischer, S. 34-39.

Müller, Adam (1936): Die Elemente der Staatskunst. Sechsunddreißig Vorlesungen [1808-1808]. Ungekürzte Ausgabe. Neuausgabe der Originalausgabe 1808-1809. Berlin: Haude \& Spener'sche Verlagsbuchhandlung.

Nassehi, Armin (2015): Die letzte Stunde der Wahrheit. Warum rechts und links keine Alternativen mehr sind und Gesellschaft ganz anders beschrieben werden muss. Hamburg: Murmann.

Nutt, Harry (2017): Der Kulturkampf der Neuen Rechten. URL: https://www.fr.de/kultur/kulturkampf-neuenrechten-11051353.html (14.09.2020).

Ong, Walter J. (1987): Oralität und Literalität. Die Technologisierung des Wortes. Opladen: Westdeutscher Verlag.

Ottmann, Henning (2010): Geschichte des politischen Denkens. Bd. 4: Das 20. Jahrhundert, Teilband I: Der Totalitarismus und seine Überwindung. StuttgartWeimar: J. B. Metzler.

Pieper, Irene (2010): Lese- und literarische Sozialisation. In: Kämper-van den Boogart, Michae/Spinner, Kaspar H. (Hg.): Lese- und Literaturunterricht. Teil 1: Geschichte und Entwicklung, konzeptionelle und empirische Grundlagen. Baltmannsweiler: Schneider, S. $87-148$. 
Rossbacher, Karlheinz (2000): Heimatkunst der frühen Moderne. In: Naturalismus, Fin de siécle, Expressionismus 1890-1918 [= Hansers Sozialgeschichte der deutschen Literatur, Band 7]. München: dtv, S. 300-314.

Salzborn, Samuel (2020): Kollektive Unschuld. Die Abwehr der Shoah im deutschen Erinnern. Leipzig: Hentrich \& Hentrich.

Safranski, Rüdiger (2017): Eine fahrlässige und hysterische Debatte. Rüdiger Safranski im Gespräch mit Joachim Scholl. URL: https:// www.deutschlandfunkkultur.de/rolf-petersieferle-und-sein-finis-germania-eine.2162. de.html?dram:article_id=389507 (11.09.2020).

Schmitt, Carl (1982): Der Leviathan in der Staatslehre des Thomas Hobbes. Sinn und Feh/schlag eines politischen Symbols [1938]. Mit einem Anhang sowie einen Nachwort des Herausgebers. Stuttgart: Klett-Cotta.

Schmitt, Carl (1984): Römischer Katholizismus und politische Form [1923]. Stuttgart: Klett-Cotta.

Schneider, Manfred (1999): Politik der Lebensgeschichte um 1800 und das autobiographische Wissen im Theoriedesign des 20. Jahrhunderts. In: Vogl, Joseph (Hg.): Poetologien des Wissens um 1800. München: Fink, S. 267-288.

Seibt, Gustav/et.al. (2017): Ein erschreckender Absturz. URL: https://www.deutschlandfunkkultur. de/sz-literaturkritiker-gustav-seibt-ueber-finisgermania-ein.1008.de.html?dram:article_id=388580; https://www.deutschlandfunkkultur.de/rolf-petersieferle-und-sein-finis-germania-eine.2162. de.html?dram:article_id=389507 (28.04.21).

Sieferle, Rolf Peter (1995): Die Konservative Revolution. Fünf biographische Skizzen. Frankfurt/M.: Fischer.

Sieferle, Rolf Peter (1997): Rückblick auf die Natur. Eine Geschichte des Menschen und seiner Umwelt. München: Beck.

Sieferle, Rolf Peter (2019): Finis Germania [2017]. Berlin: Landtverlag.

Spengler, Oswald (1998): Der Untergang des Abendlandes. Umrisse einer Morphologie der Weltgeschichte [1923]. München: C. H. Beck.

Spoerhase, Carlos (2017): Politik der Form. Autosoziobiografie als Gesellschaftsanalyse. In: Merkur: Deutsche Zeitschrift für europäisches Denken 71, Nr. 818, S. 27-37.

Stanitzek, Georg (1999): Otto Luschnat: Autodidaktos. Eine Begriffsgeschichte. In: Dotzler, Bernhard (Hg.): Grundlagen der Literaturwissenschaft: exemplarische Texte. Köln u. a.: Böhlau, S. 325- 341.

Steinmayr, Markus (2020): Fridays for Yesterday: Ein Kommentar zur rechten Ökologie. In: Merkur: Deutsche Zeitschrift für europäisches Denken 74, Nr. 855 , S. 20-31.
Steinmayr, Markus (2021): Autodidakten, Philister, Parrhesiasten. Bildungsfiguren der Neuen Rechten. In: Merkur: Deutsche Zeitschrift für europäisches Denken 74, Nr. 862, S. 77-87.

Stöckmann, Ingo (2009): Erkenntnislogik und Narrativik der Moderne. Einige Bemerkungen zur Anke-Marie Lohmeiers Aufsatz ,Was ist eigentlich ,modern?' und Thomas Anz' Kritik. In: Internationales Archiv für Sozialgeschichte der deutschen Literatur 34/1, S. 221-234.

Stöckmann, Ingo (2012): Moderne und Kultur. Über Genese und Funktionsweise literaturwissenschaftlicher Moderne-Begriffe. In: Internationales Archiv für Sozialgeschichte der deutschen Literatur 37/1, S. 105-118.

Strauß, Botho (1993): Anschwellender Bocksgesang. URL: https://www.spiegel.de/spiegel/print/d-13681004. html (28.04.21).

Thomalla, Erika/Gladić, Mladen (2021): Literatur als Klartext. Wie Rechte lesen. In: Merkur: Deutsche Zeitschrift für europäisches Denken 74, Nr. 862, S. 5-16.

Waldstein, Thor von (2019): Metapolitik. Theorie-LageAktion. Schnellroda: Antaios.

Winko, Simone (1996): Literarische Wertung und Kanonbildung. In: Arnold, Heinz Ludwig/Detering, Heinrich (Hg.): Grundzüge der Literaturwissenschaft. München: dtv, S. 585-600.

Vogl, Joseph (2021): Kapital und Ressentiment. Eine kurze Theorie der Gegenwart. München: C. H. Beck

Wildt, Michael (2019): Die Ambivalenz des Volkes. Der Nationalsozialismus als Gesellschaftsgeschichte. Berlin: Suhrkamp.

Wolf, Friedrich August (1835): Friedr. Aug. Wolf über Erziehung, Schule, Universität. Aus Wolfs literarischem Nachlasse zusammengestellt von Wilhelm Körte. Quedlinburg und Leipzig: Becker'sche Buchhandlung.

Young, Eduard (1768): Dr. Eduard Young's Klagen oder Nachtgedanken über Leben, Tod und Unsterblichkeit In neun Nächten. Nebst Desselben Charakteristischen Satire auf die Ruhmbegierde, die allgemeine Leidenschaft. Aus dem Englischen ins Deutsche übersetzt, durchgehends mit kritischen und erläuternden Anmerkungen begleitet, und mit dem nach letzten Ausgabe abgedruckten Originale herausgegeben von J. A. Ebert. Erster Band, Zweyte verbesserte Auflage. Braunschweig.

Zymner, Rüdiger (2005): Gattung. In: Schweikle, Günther/Burdorf, Dieter/u.a. (Hg.): Metzler Lexikon Literatur. 3. völlig neue bearbeitete Aufl. StuttgartWeimar: J. B. Metzler, S. 261-262. 